\title{
A NOTE ON THE ENERGY RELEASE RATE IN QUASI-STATIC ELASTIC CRACK PROPAGATION*
}

\author{
JAMES K. KNOWLES $\dagger$
}

\begin{abstract}
This paper considers analytical issues associated with the notion of the energy release rate in quasi-static elastic crack propagation.
\end{abstract}

1. Introduction. When a crack in a deformed elastic solid lengthens, the new free surface created requires energy for its formation. If the crack propagation process is slow enough to be treated as quasi-static, ${ }^{1}$ continuum mechanical models permit the calculation of the rate at which energy is made available for the generation of new free surface in terms of the solution of the governing equilibrium problem corresponding to the deformation of the cracked solid under given loads.

The essential physical ideas involved in this calculation, as well as the resulting formula, have been known for some time. One may refer, for example, to the work of Atkinson and Eshelby [1], Budiansky and Rice [2], Eshelby [3], [4], Rice [5] and Sanders [6]. To make clear the most appropriate underlying mathematical assumptions, however, seems to be more troublesome. Gurtin [7] has recently undertaken to give a precise analysis in such a way as to be valid for two-dimensional problems in nonlinear as well as linear elasticity. His arguments, however, rely on a relatively large number of assumptions, some of which pertain to properties of the solution to the relevant boundary value problem which-ideally, at least-should be deduced, rather than assumed.

In the present note, we consider the simplest possible case of quasi-static elastic crack propagation in order to illustrate and clarify the purely analytical issues involved. We treat the crack problem in anti-plane shear ${ }^{2}$ according to the linearized theory of elasticity. The argument given here is entirely different from, and involves substantially fewer assumptions than, that given in [7].

2. The crack problem. We consider a homogeneous, isotropic elastic cylinder in an equilibrium state of anti-plane shear. The outer boundary of a cross-section of the cylinder is a piecewise smooth simple closed curve $C$, while the inner boundary consists of a line segment of length $l$ (the crack). With Cartesian coordinates chosen as in Fig. 1, the crack is described by $x_{2}=0,0 \leqq x_{1} \leqq l$. We denote by $\mathscr{R}(l)$ the region consisting of points on or inside $C$ which do not lie on the crack.

According to the linearized theory of elasticity, the out-of-plane displacement $u=u\left(x_{1}, x_{2}\right)$ of particles in the cross-section of the cylinder is a harmonic function:

$$
\Delta u \equiv u, 11+u, 22=0 \quad \text { on } \mathscr{R}(l) .
$$

* Received by the editors September 3, 1980, and in final form December 9, 1980. The results communicated in this paper were obtained in the course of an investigation supported in part by Contract N00014-75-C-0196 with the Office of Naval Research in Washington, D.C.

$\dagger$ Division of Engineering and Applied Science, California Institute of Technology, Pasadena, California 91125.

${ }^{1}$ That is, if inertia effects can be neglected.

${ }^{2}$ Anti-plane shear refers to the class of deformations of a cylinder in which the displacement vector is parallel to the generators of the cylinder and independent of axial position. For a discussion of anti-plane shear (or "Mode III" displacement fie! "s) in the theory of elasticity, see [8]. 


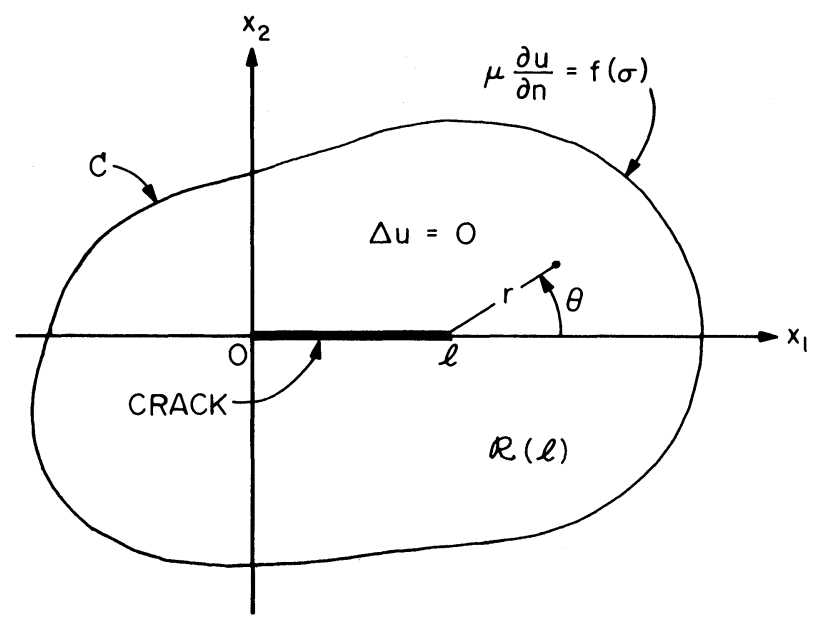

Fig. 1. Cross-section of cracked cylinder.

Here a comma followed by a subscript indicates partial differentiation with respect to the corresponding Cartesian coordinate. On the outer boundary,

$$
\mu \frac{\partial u}{\partial n}=f(\sigma) \quad \text { on } C
$$

where the constant $\mu>0$ is the shear modulus of the elastic material, $f$ represents the given traction on $C$, and $\sigma$ is arc length, measured positive counterclockwis $\%$ The derivative in (2.2) is in the direction of the unit outward normal vector $\mathbf{n}$ on $C$. The faces of the crack are to be traction-free, so that

$$
u, 2=0 \quad \text { at } x_{2}=0 \pm, \quad 0<x_{1}<l .
$$

Further,

$$
u \text { is bounded on } \mathscr{R}(l) \text {. }
$$

In the model of quasi-static crack propagation envisaged here, the crack lengthens in time by propagating to the right only; $l$ is taken to be a strictly increasing function of the time. Without loss of generality, we may then in fact identify $l$ with the time.

We assume the existence of a solution $u=u(\mathbf{x} ; l)=u\left(x_{1}, x_{2} ; l\right)$ to the problem (2.1) $-(2.4)$ which is defined and three times continuously differentiable with respect to $(\mathbf{x}, l)$ on the three-dimensional region characterized by $\mathbf{x} \in \mathscr{R}(l), 0<l \leqq l_{0}$, for some fixed, positive $l_{0}$ such that the point $\left(l_{0}, 0\right)$ lies inside $C$. It is further assumed that $u$ and all of its derivatives of order up to and including three possess limits as a point $\left(x_{1}, 0\right)$ on the crack is approached from above, provided $0<x_{1}<l$. These limits are assumed to be continuous functions of $\left(x_{1}, l\right)$ for $0<x_{1}<l, 0<l \leqq l_{0}$. Finally, we make analogous assumptions for the corresponding limits as the crack is approached from below. ${ }^{3}$

Except for cases corresponding to very special choices of the loading $f, \nabla u$ is unbounded at both crack-tips.

\footnotetext{
${ }^{3}$ Our assumptions clearly impose restrictions on the given traction $f$. We leave aside the question of determining conditions on $f$ and $C$ which assure the existence of a solution $u(\mathbf{x} ; l)$ of the assumed smoothness.
} 
We shall first prove that the energy in $\mathscr{R}(l)$ associated with $u$, defined by

$$
E(l)=\int_{\mathscr{R}(l)} \frac{\mu}{2}|\nabla u|^{2} d A, \quad 0<l \leqq l_{0},
$$

is finite for each $l$, and satisfies

$$
2 E(l)=\int_{C} f(\sigma) u(\mathbf{x} ; l) d \sigma, \quad 0<l \leqq l_{0} .
$$

It follows immediately from (2.6) that there is at most one solution of the prescribed smoothness to the boundary value problem (2.1)-(2.4), apart from an arbitrary additive constant. The boundedness condition (2.4), of course, plays a central role in the argument leading to (2.6) and the resulting uniqueness.

Our objective is to study the so-called "energy release rate" $\mathscr{E}(l)$ defined by

$$
\mathscr{E}(l)=\int_{C} f(\sigma) \dot{u}(\mathbf{x} ; l) d \sigma-\dot{E}(l), \quad 0<l \leqq l_{0},
$$

where the dot indicates differentiation with respect to $l$. In particular,

$$
\dot{u}(\mathbf{x} ; l) \equiv \frac{\partial u}{\partial l}(\mathbf{x} ; l) .
$$

$\mathscr{E}(l)$ represents the excess of the power supplied by the external traction over the rate of increase of stored energy, and thus may be interpreted as the rate at which energy is made available for the formation of new free surface during the process of crack propagation. Using only the assumptions stated above, ${ }^{4}$ we shall prove that

$$
\mathscr{E}(l)=\lim _{r \rightarrow 0} \int_{\Gamma_{r}}\left(\frac{\mu}{2}|\nabla u|^{2} n_{1}-\mu \frac{\partial u}{\partial n} u, 1\right) d s,
$$

where $\Gamma_{r}$ is a circle of radius $r$ centered at the moving crack-tip $x_{1}=l, x_{2}=0, s$ is arc length on $\Gamma_{r}$ (increasing counterclockwise), and $\mathbf{n}$ is the unit normal vector on $\Gamma_{r}$ which points away from the crack-tip; $n_{1}$ is the $x_{1}$-component of $\mathbf{n}$. Thus $\mathscr{E}(l)$ depends only on the local behavior of $\nabla u$ near the moving crack-tip (and not directly on $\dot{u}$ ). We shall also prove that $\mathscr{E}(l) \geqq 0$.

In order to establish these results, we need some estimates pertaining to the behavior of $u$ and $\dot{u}$ near the crack-tips. In this connection it is necessary to observe first that $\dot{u}$ satisfies

$$
\begin{array}{ll}
\Delta \dot{u}=0 \quad \text { on } \mathscr{R}(l), \\
\frac{\partial \dot{u}}{\partial n}=0 \quad \text { on } C, \\
\dot{u}, 2_{2}=0 \quad \text { at } x_{2}=0 \pm, \quad 0<x_{1}<l .
\end{array}
$$

These follow from (2.1)-(2.3) by differentiation with respect to $l$, provided use is made of the fact that the traction $f$ is independent of $l$. If $\dot{u}$ were bounded at both crack-tips (and, therefore on $\mathscr{R}(l)$ ), the uniqueness result alluded to above would imply that $\dot{u} \equiv$ constant on $\mathscr{R}(l)$. It will turn out, however, that $\dot{u}$ is in general unbounded near the moving crack-tip.

We now consider the local behavior of harmonic functions near a crack tip.

\footnotetext{
${ }^{4}$ In particular, we make no assumptions about $\dot{u}$ beyond those stated in the smoothness hypotheses described above.
} 
3. Crack-tip estimates. Let $\mathscr{D}$ be the plane slit disk described in polar coordinates by

$$
\mathscr{D}=\{(r, \theta) \mid 0<r \leqq a,-\pi<\theta<\pi\} .
$$

Further, let $\Gamma_{r}, 0<r \leqq a$, at present be the circle of radius $a$ centered at the origin, and denote by $\mathscr{D}_{r}, 0<r \leqq a$, and $\mathscr{B}_{r}, 0<r<a$, the sets of those points in $\mathscr{D}$ which lie respectively inside and outside $\Gamma_{r}$.

Let $\varphi=\varphi(r, \theta)$ be a function defined on $\mathscr{D}$ with the following properties:

A. $\varphi \in \mathscr{C}^{2}(\mathscr{D})$, and $\varphi$ and its derivatives ${ }^{5} \varphi_{r}$ and $\varphi_{\theta}$ possess limits as $\theta \rightarrow \pm \pi$, $0<r \leqq a$; these limits are continuous functions of $r, 0<r \leqq a$.

B. $\varphi$ is mean-square bounded on $\mathscr{D}$; i.e., there is a constant $m \geqq 0$ such that

$$
\frac{1}{2 \pi r} \int_{\Gamma_{r}} \varphi^{2} d s \leqq m^{2}, \quad 0<r \leqq a
$$

C.

$$
\varphi_{r r}+\frac{1}{r} \varphi_{r}+\frac{1}{r^{2}} \varphi_{\theta \theta}=0 \quad \text { on } \mathscr{D}
$$

D.

$$
\varphi_{\theta}(r, \pm \pi)=0, \quad 0<r \leqq a .
$$

We note that $\varphi$ has property B if $\varphi$ is bounded on $\mathscr{D}$.

The principal conclusions concerning functions with properties A-D relate to the energy distribution associated with $\varphi$ and form the basis of the analysis to follow.

The energy $E(r)$ in $\mathscr{D}_{r}$ associated with $\varphi$, defined by

$$
E(r)=\int_{\mathscr{D}_{r}} \frac{\mu}{2}|\nabla \varphi|^{2} d A, \quad 0<r \leqq a,
$$

is finite and satisfies

$$
E(r)=\int_{\Gamma_{r}} \frac{\mu}{2} \varphi_{r} \varphi d s \geqq 0, \quad 0<r \leqq a .
$$

Moreover,

$$
E(r) \rightarrow 0 \quad \text { as } r \rightarrow 0 .
$$

To prove this proposition, ${ }^{6}$ we begin by setting

$$
F(r)=\int_{\mathscr{B}_{r}} \frac{\mu}{2}|\nabla \varphi|^{2} d A, \quad 0<r<a .
$$

A standard application of the divergence theorem, together with (3.3) and (3.4), leads to

$$
F(r)=g(a)-g(r)
$$

where

$$
g(r)=\int_{\Gamma_{r}} \frac{\mu}{2} \varphi_{r} \varphi d s, \quad 0<r \leqq a
$$

\footnotetext{
${ }^{5}$ Derivatives with respect to $r$ or $\theta$ are indicated by subscripts without commas.

${ }^{6}$ The argument that follows is essentially the same as that used in a more general setting in [9].
} 
Moreover, from (3.9), (3.8),

$$
g^{\prime}(r)=-F^{\prime}(r)=\int_{\Gamma_{r}} \frac{\mu}{2}|\nabla \varphi|^{2} d s \geqq 0,
$$

so that $g(r)$ is monotone nondecreasing. It follows that $-\infty \leqq g(0+)<\infty$. From (3.10), (3.11) and Schwarz's inequality, one obtains

$$
g^{2}(r) \leqq \frac{\mu^{2}}{4} \int_{\Gamma_{r}} \varphi_{r}^{2} d s \int_{\Gamma_{r}} \varphi^{2} d s \leqq \frac{\mu}{2} g^{\prime}(r) \int_{\Gamma_{r}} \varphi^{2} d s .
$$

By (3.2) (property B),

$$
g^{2}(r) \leqq \pi \mu m^{2} r g^{\prime}(r), \quad 0<r \leqq a .
$$

Suppose first that $g(0+)<0$. Then, for some $r_{2}, 0<r_{2}<a$, one has $g(r)<0$ for $0<r \leqq r_{2}$. Integrate (3.13) from $r_{1}$ to $r_{2}$, where $0<r_{1}<r_{2}$, obtaining

$$
\log \frac{r_{2}}{r_{1}} \leqq \pi \mu m^{2}\left[\frac{1}{g\left(r_{1}\right)}-\frac{1}{g\left(r_{2}\right)}\right] \leqq \frac{\pi \mu m^{2}}{\left|g\left(r_{2}\right)\right|},
$$

or

$$
\left|g\left(r_{2}\right)\right| \leqq \frac{\pi \mu m^{2}}{\log \left(r_{2} / r_{1}\right)}
$$

Let $r_{1} \rightarrow 0$ to get $g\left(r_{2}\right)=0$, which is a contradiction. Next suppose that $g(0+)>0$. Then $g(r)>0$ for $0<r \leqq r_{2}$ for some $r_{2}, 0<r_{2}<a$. Integration of (3.13) now gives

$$
g\left(r_{1}\right) \leqq \frac{\pi \mu m^{2}}{\log \left(r_{2} / r_{1}\right)}, \quad 0<r_{1}<r_{2},
$$

from which we conclude $g(0+)=0$, again a contradiction. Thus

$$
g(r) \rightarrow 0 \quad \text { as } r \rightarrow 0 \text {. }
$$

From (3.17), (3.9), it follows that $F(0+)=g(a)$ is finite. But (see (3.5), (3.8), (3.9)),

$$
E(r)=F(0+)-F(r)=g(r), \quad 0<r \leqq a ;
$$

(3.18), (3.10) and (3.17) establish (3.6), (3.7) and the proof is complete.

4. Properties of $u, \dot{u}$ near the crack-tips. Returning to the original boundary value problem (2.1)-(2.4), we suppose first that $\bar{l}$ is a fixed but arbitrary value of $l$ with $0<\bar{l}<l_{0}$. Let $a$ be a constant such that $0<\bar{l}-2 a<\bar{l}+2 a<l_{0}$, denote by $\Lambda$ the $l$-interval $[\bar{l}-a, \bar{l}+a]$, and note that the (moving) circle $\Gamma_{a}=\Gamma_{a}(l)$ of radius $a$ centered at the right crack-tip lies in the interior of the boundary curve $C$ and never encloses the left crack-tip, provided l lies in $\Lambda$. In this section we shall consider the solution $u(\mathbf{x} ; l)$ and its derivative $\dot{u}(\mathbf{x}, l)$ only for values of $l$ in the interval $\Lambda$.

With $a$ as above, let $\Gamma_{r}=\Gamma_{r}(l) 0<r \leqq a$, be the circle of radius $r$ centered at $x_{1}=l$, $x_{2}=0$, and let $\mathscr{D}$ stand for the set described by (3.1), where $r, \theta$ are now (moving) polar coordinates centered at the right crack-tip. As in $\S 3, \mathscr{D}_{r}$ denotes the set of those points in $\mathscr{D}$ which lie inside $\Gamma_{r}, 0<r<a$. Define

$$
v(r, \theta ; l)=u(l+r \cos \theta, r \sin \theta ; l), \quad 0<r \leqq a, \quad-\pi \leqq \theta \leqq \pi, \quad l \in \Lambda,
$$

and note that

$$
\dot{v}(r, \theta ; l) \equiv \frac{\partial v}{\partial l}(r, \theta ; l)=u,_{1}+\dot{u}
$$


In view of the assumptions made on $u$ in $\S 2, v$ clearly has properties A-D for each $l$ in $\Lambda$; in particular, by $(2.4)$, there is a constant $m(l)$ such that

$$
\frac{1}{2 \pi r} \int_{\Gamma_{r}} v^{2}(r, \theta ; l) d s \leqq m^{2}(l), \quad 0<r \leqq a, \quad l \in \Lambda .
$$

In view of the proposition in $\S 3$ concerning functions with properties $A-D$, it follows from (3.7), (3.5) with $\varphi(r, \theta)=v(r, \theta ; l)$ that

$$
\int_{\mathscr{D}_{r}}|\nabla v|^{2} d A \rightarrow 0 \quad \text { as } r \rightarrow 0, \quad l \in \Lambda .
$$

From (4.2) and the fact that $u$ and $\dot{u}$ are harmonic in $\mathscr{D}$ and have vanishing first normal derivatives on the crack-faces (see (2.1), (2.3), (2.9), (2.11)), it follows that $\dot{v}$ has properties A, C, D, for each $l$ in $\Lambda$. We show next that $\dot{v}$ has property $\mathrm{B}$ (see (3.2)) for each such $l$. To this end, let $l$ and $l^{\prime}$ belong to $\Lambda$, with $l^{\prime} \neq l$. Define

$$
V\left(r, \theta ; l, l^{\prime}\right)=\frac{v(r, \theta ; l)-v\left(r, \theta ; l^{\prime}\right)}{l-l^{\prime}}, \quad 0<r \leqq a, \quad-\pi \leqq \theta \leqq \pi, \quad l, l^{\prime} \in \Lambda, \quad l^{\prime} \neq l
$$

Clearly $V$ has properties A-D and hence, choosing $\varphi=V$ in (3.6) and making use of (3.7), one has

$$
\int_{\Gamma_{r}} V V_{r} d s \geqq 0, \quad 0<r \leqq a, \quad l, l^{\prime} \in \Lambda, \quad l \neq l^{\prime} .
$$

The idea now is to let $l^{\prime} \rightarrow l$ with $r$ fixed in (4.6). To carry out this limit process, we first observe from (4.5) that

$$
V\left(r, \theta ; l, l^{\prime}\right) \rightarrow \dot{v}(r, \theta ; l) \quad \text { as } l^{\prime} \rightarrow l, \quad \text { for each fixed }(r, \theta) .
$$

Moreover, from (4.5), the mean value theorem and the smoothness properties that $v$ inherits from $u$ through (4.1), it follows that

$$
\left|V\left(r, \theta ; l, l^{\prime}\right)\right| \leqq M_{1}(r), \quad 0<r \leqq a, \quad-\pi \leqq \theta \leqq \pi, \quad l^{\prime}, l \in \Lambda, \quad l^{\prime} \neq l,
$$

and

$$
\left|V_{r}\left(r, \theta ; l, l^{\prime}\right)\right| \leqq M_{2}(r), \quad 0<r \leqq a, \quad-\pi \leqq \theta \leqq \pi, \quad l^{\prime}, l \in \Lambda, \quad l^{\prime} \neq l,
$$

where

(4.10) $M_{1}(r)=\max _{\substack{-\pi \leqq \theta \leqq \pi \\ \lambda \in \Lambda}}|\dot{v}(r, \theta ; \lambda)|, \quad M_{2}(r)=\max _{\substack{-\pi \leqq \theta \leqq \pi \\ \lambda \in \Lambda}}\left|\dot{v}_{r}(r, \theta ; \lambda)\right|, \quad 0<r \leqq a$.

It follows that

$$
\left|V V_{r}\right| \leqq M_{1} M_{2} \quad \text { on } \Gamma_{r}, \quad 0<r \leqq a, \quad l^{\prime}, l \in \Lambda, \quad l^{\prime} \neq l .
$$

Thus the pointwise convergence in (4.7), although not necessarily uniform, is dominated. It follows from a standard theorem ${ }^{8}$ that the limits as $l^{\prime} \rightarrow l$ may be taken inside the integral in (4.6), yielding

$$
\int_{\Gamma_{r}} \dot{v} \dot{v}_{r} d s \geqq 0, \quad 0<r \leqq a, \quad l \in \Lambda .
$$

\footnotetext{
${ }^{7}$ In fact, $|v| \leqq m$ on $\mathscr{D}$.

${ }^{8}$ See $[10$, p. 405].
} 
Thus,

$$
\frac{d}{d r}\left(\int_{\Gamma_{r}} \frac{\dot{v}^{2}}{r} d s\right) \geqq 0, \quad 0<r \leqq a, \quad l \in \Lambda,
$$

so that the integral in (4.13) is a nonnegative, monotone nondecreasing function of $r$. It follows that this integral is a bounded function of $r, 0<r \leqq a$; i.e., there is a constant $n(l) \geqq 0$ such that

$$
\frac{1}{2 \pi r} \int_{\Gamma_{r}} \dot{v}^{2} d s \leqq n^{2}(l), \quad 0<r \leqq a, \quad l \in \Lambda .
$$

Thus $\dot{v}$ has property $\mathrm{B}$ for each $l$ in $\Lambda$. The main proposition of $\S 3$ may now be invoked with $\varphi=\dot{v}$; from (3.6), (3.7) we find that

$$
\int_{\mathscr{D}_{r}}|\nabla \dot{v}|^{2} d A \rightarrow 0 \quad \text { as } r \rightarrow 0, \quad l \in \Lambda .
$$

We note that (4.3), (4.14) imply

$$
\int_{\Gamma_{r}} v^{2} d s=O(r), \quad \int_{\Gamma_{r}} \dot{v}^{2} d s=O(r) \quad \text { as } v \rightarrow 0, \quad l \in \Lambda .
$$

Finally, we observe that, since the choice of the center $\bar{l}$ of the interval $\Lambda$ was arbitrary, each of the four limiting results (4.4), (4.15), (4.16) holds for every $l$ in $\left(0, l_{0}\right)$.

It may be remarked that a more refined argument, using more detailed properties of $v$, can be used to prove that $\dot{v}$ is actually continuous at the moving crack-tip, ${ }^{9}$ and not merely mean-square bounded as asserted in (4.14). Since (4.14) is sufficient for our purposes, we omit the proof of the continuity of $\dot{v}$.

As remarked earlier, $\nabla u$ is in general not bounded-or even mean-square bounded-near a crack tip. According to (4.2) and (4.14), therefore, $\dot{u}$ would in general not be bounded near the moving crack-tip.

Analysis parallel to that given above can be carried out near the left (fixed) crack-tip. One sets

$$
w(r, \theta ; l)=u(r \cos \theta, r \sin \theta ; l),
$$

where $r, \theta$ are now polar coordinates centered at the origin. In contrast to (4.2), we have

$$
\dot{w}=\dot{u} .
$$

If $\Gamma_{r}$ is a circle of radius $r$ centered at the origin, while $\mathscr{D}_{r}$ is the interior of $\Gamma_{r}$ with the crack deleted, one can again show that

$$
\int_{\Gamma_{r}} w^{2} d s=O(r), \quad \int_{\Gamma_{r}} \dot{w}^{2} d s=O(r) \quad \text { as } r \rightarrow 0, \quad 0<l<l_{0},
$$

as well as

$$
\int_{\mathscr{D}_{r}}|\nabla w|^{2} d A \rightarrow 0, \quad \int_{\mathscr{D}_{r}}|\nabla \dot{w}|^{2} d A \rightarrow 0 \quad \text { as } r \rightarrow 0, \quad 0<l<l_{0} .
$$

We omit the details.

\footnotetext{
${ }^{9}$ Continuity of the analogous physical quantity in plane strain at the moving crack-tip is assumed in [7].

${ }^{10}$ Again, $\dot{w}$ can be shown to be continuous, and not merely mean-square bounded, at the left crack-tip.
} 
5. Energy release rate. It is clear from the local considerations of the preceding section that the energy $E(l)$ of $(2.5)$ associated with the boundary value problem $(2.1)-(2.4)$ is finite. In order to study $E(l)$, we let $\Gamma_{r}^{(1)}, \Gamma_{r}^{(2)}$ be circles of radius $r$, $0<r \leqq a$, centered at the left and right crack-tips, respectively. ${ }^{11}$ Let $\mathscr{R}_{r}(l)$ be the set of those points which lie in $\mathscr{R}(l)$ but outside $\Gamma_{r}^{(1)}+\Gamma_{r}^{(2)}, 0<r \leqq a$. An application of the divergence theorem to the integral of $(\mu / 2)|\nabla u|^{2}$ over $\mathscr{R}_{r}(l)$, accounting for the presence of the crack and making use of (2.1), (2.3), followed by the limit $r \rightarrow 0$, leads easily to the conclusion that

$$
E(l)=\frac{1}{2} \int_{C} f(\sigma) u(\mathbf{x} ; l) d \sigma, \quad 0<l \leqq l_{0},
$$

as claimed in (2.6).

Since the given traction $f$ on $C$ is independent of $l$, it follows immediately from (5.1) that

$$
2 \dot{E}(l)=\int_{C} f(\sigma) \dot{u}(\mathbf{x} ; l) d \sigma .
$$

An alternative expression for $\dot{E}(l)$ may be obtained from (5.2) by proceeding as follows. From (5.2), (2.2),

$$
\begin{aligned}
2 \dot{E}(l) & =\int_{C} \mu \frac{\partial u}{\partial n} \dot{u} d \sigma \\
& =\int_{S_{r}} \mu \frac{\partial u}{\partial n} \dot{u} d s-\int_{\Gamma_{r}^{(1)}} \mu \frac{\partial u}{\partial n} \dot{u} d s-\int_{\Gamma_{r}^{(2)}} \mu \frac{\partial u}{\partial n} \dot{u} d s,
\end{aligned}
$$

where $S_{r}=C+\Gamma_{r}^{(1)}+\Gamma_{r}^{(2)}$, and $\mathbf{n}$ is the unit normal on $S_{r}$, taken outward with respect to $\mathscr{R}_{r}(l)$. The arc lengths and integrals over $\Gamma_{r}^{(1)}, \Gamma_{r}^{(2)}$ are taken counterclockwise. The divergence theorem, together with the facts that $u$ and $\dot{u}$ are harmonic and satisfy the free surface conditions (2.3) and (2.12) on the crack faces, permits one to write

$$
\int_{S_{r}} \mu \frac{\partial u}{\partial n} \dot{u} d s=\int_{S_{r}} \mu \frac{\partial \dot{u}}{\partial n} u d s .
$$

Making use of (2.11) in (5.4), we find that

$$
\int_{S_{r}} \mu \frac{\partial u}{\partial n} \dot{u} d s=\int_{\Gamma_{r}^{(1)}} \mu \frac{\partial \dot{u}}{\partial n} u d s+\int_{\Gamma_{r}^{(2)}} \mu \frac{\partial \dot{u}}{\partial n} u d s,
$$

so that (5.3) may be written in the form

$$
2 \dot{E}(l)=I_{1}(r ; l)+I_{2}(r ; l),
$$

where

$$
I_{\alpha}(r ; l)=\int_{\Gamma_{r}^{(\alpha)}} \mu\left(\frac{\partial \dot{u}}{\partial n} u-\frac{\partial u}{\partial n} \dot{u}\right) d s, \quad \alpha=1,2 .
$$

Equations (5.6), (5.7) show that $\dot{E}(l)$ depends only on the behavior of $u$ and $\dot{u}$ near the crack-tips.

\footnotetext{
${ }^{11}$ Here $a$ is assumed small emough to $\operatorname{keep} \Gamma_{a}^{(1)}$ and $\Gamma_{a}^{(2)}$ inside $C$ and to assure that each circle encloses only one crack-tip.

${ }^{12}$ The representation (5.6), (5.7) for $E(l)$ is the analog in anti-plane shear of Sanders' $I$-integral in plane strain or plane stress (see Eq. (4) of [6]).
} 
Consider first the integral $I_{2}$ : from (4.1) and (4.2),

$$
I_{2}=2 J+H_{2} \text {, }
$$

where

$$
\begin{gathered}
J=J(r ; l)=\int_{\Gamma_{r}^{(2)}} \frac{\mu}{2}\left[\frac{\partial u}{\partial n} u, 1-\frac{\partial}{\partial n}(u, 1) u\right] d s, \\
H_{2}=H_{2}(r ; l)=\int_{\Gamma_{r}^{(2)}} \mu\left(v_{r} \dot{v}-\dot{v}_{r} v\right) d s .
\end{gathered}
$$

A direct calculation which makes use of the facts that $v, \dot{v}$ are harmonic and $v_{\theta}, \dot{v}_{\theta}$ vanish at $\theta= \pm \pi$ reveals that $\partial H_{2} / \partial r=0$, so that the integral in (5.10) is independent of $r: H_{2}(r ; l)=H_{2}(l)$. If one now integrates $(5.10)$ with respect to $r$ over the interval $0<r<r_{1}, r_{1}<a$, one finds

$$
r_{1} H_{2}(l)=\int_{\mathscr{D}_{r_{1}}} \mu\left(v_{r} \dot{v}-\dot{v}_{r} v\right) d A .
$$

The Schwarz inequality now gives

$$
r_{1}^{2} H_{2}^{2}(l) \leqq 2 \mu^{2}\left\{\int_{\mathscr{D}_{r_{1}}} v_{r}^{2} d A \int_{\mathscr{D}_{r_{1}}} \dot{v}^{2} d A+\int_{\mathscr{D}_{r_{1}}} \dot{v}_{r}^{2} d A \int_{\mathscr{D}_{r_{1}}} v^{2} d A\right\}
$$

From (4.14) and (4.3) one finds that

$$
\int_{\mathscr{D}_{r_{1}}} v^{2} d A \leqq \pi m^{2}(l) r_{1}^{2}, \quad \int_{\mathscr{D}_{r_{1}}} \dot{v}^{2} d A \leqq \pi n^{2}(l) r_{1}^{2},
$$

so that (5.12) yields

$$
H_{2}^{2}(l) \leqq 2 \pi \mu^{2}\left[m^{2}(l) \int_{\mathscr{D}_{r_{1}}}|\nabla \dot{v}|^{2} d A+n^{2}(l) \int_{\mathscr{D}_{r_{1}}}|\nabla v|^{2} d A\right] .
$$

Letting $r_{1} \rightarrow 0$ in (5.14) and using (4.15), (4.4), we find

$$
\mathrm{H}_{2}(l)=0 \text {, }
$$

so that, from (5.8),

$$
\lim _{r \rightarrow 0} I_{2}(r ; l)=\lim _{r \rightarrow 0} J(r ; l) .
$$

As to the integral $I_{1}(r, l)$, one shows by a similar argument based on (4.18), (4.19) and (4.20) that

$$
\lim _{r \rightarrow 0} I_{1}(r ; l)=0 \text {. }
$$

Letting $r \rightarrow 0$ in (5.6) and using (5.16), (5.17), and (5.8), we obtain

$$
\dot{E}(l)=\lim _{r \rightarrow 0} J(r ; l) .
$$

where $J$ is given by (5.9). Note that $J$ does not involve $\dot{u}$ and depends only on the behavior of $u$ near the moving crack-tip.

\footnotetext{
${ }^{13}$ The finiteness of the integrals on the right in (5.12) assures the existence of the integral in (5.11).
} 
A more convenient representation for $J$ can be found as follows. Since by (4.1)

$$
u, 1=v_{r} \cos \theta-\frac{1}{r} v_{\theta} \sin \theta
$$

one may write (5.9) as

$$
J=-\frac{\mu}{2} \int_{\Gamma_{r}^{(2)}}\left[v_{r}\left(v_{r} \cos \theta-\frac{1}{r} v_{\theta} \sin \theta\right)-v \frac{\partial}{\partial r}\left(v_{r} \cos \theta-\frac{1}{r} v_{\theta} \sin \theta\right)\right] d s .
$$

The fact that $v$ is harmonic can be used to eliminate $v_{r r}$ from (5.20); integrations by parts and the boundary conditions $v_{\theta}=0$ at $\theta= \pm \pi$ then give

$$
J=\int_{\Gamma_{r}^{(2)}} \mu\left(\frac{1}{2}|\nabla u|^{2} n_{1}-u, \frac{\partial u}{\partial n}\right) d s .
$$

Here $n$ is the unit normal on $\Gamma_{r}^{(2)}$ pointing away from the crack-tip, and $n_{1}=\cos \theta$ is its $x_{1}$-component. The integral in $(5.21)$ is the usual $J$-integral of fracture mechanics discovered by Eshelby [4] and independently by Rice [11]. As these authors have shown, and as is easily verified, $J$ is independent of $r$. In fact, the integral

$$
J=J(l)=\int_{\Gamma} \mu\left(\frac{1}{2}|\nabla u|^{2} n_{1}-u, 1 \frac{\partial u}{\partial n}\right) d s
$$

is independent of the path $\Gamma$, as long as $\Gamma$ is a curve in $\mathscr{R}(l)$ which begins and ends on the faces of the crack and surrounds the right crack-tip but not the left one. Thus (5.18) may be replaced by

$$
\dot{E}(l)=J(l),
$$

with $J(l)$ given by (5.22). In view of (5.2), (5.23) the energy release rate of (2.7) is given by

$$
\mathscr{E}(l)=\dot{E}(l)=J(l)
$$

Thus the power delivered by the external load is divided equally between the tasks of increasing the stored energy and making energy available for crack propagation.

Equations (5.24), (5.22) show that $\mathscr{E}(l)$ (and $\dot{E}(l)$ ) depends only on the local behavior of $\nabla u$ near the moving crack-tip.

In order to show that $\mathscr{E}(l) \geqq 0$, it is convenient to introduce a function $\mathscr{P}\left(l, l^{\prime}\right)$ as follows: for $0<l^{\prime} \leqq l<l_{0}$, set

$$
\mathscr{P}\left(l, l^{\prime}\right)=\int_{\mathscr{R}(l)} \frac{\mu}{2}\left|\nabla u\left(\mathbf{x}, l^{\prime}\right)\right|^{2} d A-\int_{C} f(\sigma) u\left(\mathbf{x}, l^{\prime}\right) d \sigma,
$$

and let

$$
P(l)=\mathscr{P}(l, l) ;
$$

$P(l)$ is called the potential energy ${ }^{14}$ in $\mathscr{R}(l)$ associated with $u$. Moreover, (5.1), (2.5) give

$$
P(l)=-E(l),
$$

so that

$$
\dot{P}(l)=-\dot{E}(l)
$$

\footnotetext{
${ }^{14}$ It follows from properties of $u(\mathbf{x}, l)$ already established that $\mathscr{P}\left(l, l^{\prime}\right), P(l)$ are finite.
} 
Let $\Gamma_{r}^{(1)}, \Gamma_{r}^{(2)}$ be circles of radius $r$ centered at $(0,0)$ and $(l, 0)$ as before, and let $\Gamma_{r}^{(3)}$ be a circle of radius $r$ centered at $\left(l^{\prime}, 0\right)$, where $0<l^{\prime}<l$. Let $\mathscr{R}_{r}\left(l, l^{\prime}\right)$ be the set of those points in $\mathscr{R}(l)$ which lie outside $\Gamma_{r}^{(1)}+\Gamma_{r}^{(2)}+\Gamma_{r}^{(3)}$. Set

$$
\mathscr{P}_{r}\left(l, l^{\prime}\right)=\int_{\mathscr{R}_{r}\left(l, l^{\prime}\right)} \frac{\mu}{2}\left|\nabla\left(\mathbf{x}, l^{\prime}\right)\right|^{2} d A-\int_{C} f(\sigma) u\left(\mathbf{x}, l^{\prime}\right) d \sigma, \quad 0<l^{\prime}<l<l_{0}
$$

The divergence theorem and (2.1)-(2.3) make it possible to show that

$$
\begin{aligned}
\mathscr{P}_{r}\left(l, l^{\prime}\right)-\mathscr{P}_{r}(l, l) & =\int_{\mathscr{R}_{r}\left(l, l^{\prime}\right)} \frac{\mu}{2}\left|\nabla u(\mathbf{x}, l)-\nabla u\left(\mathbf{x}, l^{\prime}\right)\right|^{2} d A+Q_{r}\left(l, l^{\prime}\right) \\
& \geqq Q_{r}\left(l, l^{\prime}\right),
\end{aligned}
$$

where

$$
Q_{r}\left(l, l^{\prime}\right)=\int_{\Gamma_{r}^{(1)}+\Gamma_{r}^{(2)}+\Gamma_{r}^{(3)}}\left[u\left(\mathbf{x}, l^{\prime}\right)-u(\mathbf{x}, l)\right] \frac{\partial u}{\partial n}(\mathbf{x}, l) d s .
$$

By using the crack-tip estimates of $\S 4$, one can show that $Q_{r}\left(l, l^{\prime}\right) \rightarrow 0$ as $r \rightarrow 0$. Letting $r \rightarrow 0$ in (5.30) and noting $\mathscr{P}_{r}\left(l, l^{\prime}\right) \rightarrow \mathscr{P}\left(l, l^{\prime}\right)$ as $r \rightarrow 0$, we obtain

$$
\mathscr{P}\left(l, l^{\prime}\right) \geqq \mathscr{P}(l, l)=P(l), \quad 0<l^{\prime}<l .
$$

But

$$
\int_{\mathscr{R}(l)} \frac{1}{2}\left|\nabla u\left(\mathbf{x}, l^{\prime}\right)\right|^{2} d A=\int_{\mathscr{R}\left(l^{\prime}\right)} \frac{1}{2}\left|\nabla u\left(\mathbf{x}, l^{\prime}\right)\right|^{2} d A,
$$

so from (5.25), (5.26), (5.33),

$$
\mathscr{P}\left(l, l^{\prime}\right)=\mathscr{P}\left(l^{\prime}, l^{\prime}\right)=P\left(l^{\prime}\right), \quad 0<l^{\prime}<l .
$$

Combining (5.34), (5.32) gives

$$
P\left(l^{\prime}\right) \geqq P(l), \quad 0<l^{\prime}<l,
$$

so that $P(l)$ is nonincreasing with $l$. Thus from (5.28), $\dot{E}(l) \geqq 0$, and from (5.24), $\mathscr{E}(l) \geqq 0$.

The inequality (5.35) may be viewed as an instance of the minimum principle associated with the boundary value problem (2.1)-(2.4) (the principle of minimum potential energy).

Acknowledgment. The author acknowledges many helpful discussions with Eli Sternberg.

\section{REFERENCES}

[1] C. AtKinson AND J. D. Eshelby, The flow of energy into the tip of a moving crack, Internat. J. Fracture Mech., 4 (1968), pp. 3-8.

[2] B. BudiAnSKy AND J. R. RiCE, Conservation laws and energy release rates, J. Appl. Mech., 40 (1973), pp. 201-203.

[3] J. D. EsHELBY, Energy relations and the energy-momentum tensor in continuum mechanics, Inelastic Behavior of Solids, M. F. Kanninen et al., eds., McGraw-Hill, New York, 1956. 
[4] J. D. Eshelby, The continuum theory of lattice defects, Solid State Physics, vol. 3, F. Seitz and D. Turnbull, eds., Academic Press, New York, 1956.

[5] J. R. RICE, Mathematical analysis in the mechanics of fracture, Fracture, an Advanced Treatise, vol. 2, H. Liebowitz, ed., Academic Press, New York, 1968.

[6] J. L. SANDERS, On the Griffith-Irwin fracture theory, J. Appl. Mech., 27 (1960), pp. 352-353.

[7] M. E. GURTIN, On the energy release rate in quasi-static elastic crack propagation, J. Elasticity, 9 (1979), pp. 187-195.

[8] I. N. SNeddon ANd M. Lowengrub, Crack Problems in the Classical Theory of Elasticity, John Wiley, New York, 1969.

[9] J. K. KNOWles AND T. A. PUCIK, Uniqueness for plane crack problems in linear elastostatics, J. Elasticity, 3 (1973), pp. 155-160.

[10] T. M. Apostol, Mathematical Analysis, Addison-Wesley, Reading, MA, 1957.

[11] J. R. RICE, A path-independent integral and the approximate analysis of strain concentration by notches and cracks, J. Appl. Mech., 35 (1968), p. 379. 